\title{
Some Generalization of Nearaffine Planes
}

\author{
by \\ Jan JAKÓBOWSKI \\ Presented by Jan RYCHLEWSKI
}

Summary. There are three kinds of Benz planes: Möbius planes, Laguerre planes and Minkowski planes. A Minkowski plane satisfying an additional axiom is connected with some other structure called a nearaffine plane. We construct an analogous structure for a Laguerre plane. Moreover, our description is common for both cases.

1. Introduction. In the case of a Minkowski plane $\mathcal{B}, \mathrm{H}$. A. Wilbrink [10] investigated the external structure $\mathcal{B}^{P}[4$, p. 3], where circles and generators are treated as blocks. A slight modification $\mathcal{B}[P]$ of $\mathcal{B}^{P}$ is called the residual plane in $P$. More precisely, all points lying on generators through $P$ are removed and each trace of a circle not passing through $P$ is supplemented with exactly one point. If a Minkowski plane $\mathcal{B}$ satisfies some additional condition, then the residual plane $\mathcal{B}[P]$ becomes a variation of a nearaffine plane considered before by J. André $[1,11]$. Such a nearaffine plane is a quadruple $(\Omega, \Xi, \triangleright, \equiv)$ with the point set $\Omega$, the set $\Xi$ of lines (subsets of $\Omega$ ) and an equivalence relation $\equiv \subset \Xi \times \Xi$ called parallelism of lines. Finally, the operation $\triangleright$, called join, maps the set of all ordered pairs of distinct points onto $\Xi$. So-called straight lines are distinguished in $\Xi$, the remaining lines are called proper, and $Q$ is called the base point of the line $\triangleright(Q, R)$. If a point $P$ of a Minkowski plane $\mathcal{B}$ induces a nearaffine plane $\mathcal{B}[P]$ then circles not through $P$ and generators not through $P$ become proper lines and straight lines, respectively.

We wish to consider an analogous situation for Laguerre planes. That is, we want to get a structure with points, lines, parallelism and join which (maybe only in special cases) can be obtained as the external structure with

2000 Mathematics Subject Classification: 51A15, 51A45, 51B15, 51B20.

Key words and phrases: affine plane, external structure, Laguerre plane, Minkowski plane, nearaffine plane. 
respect to some fixed point $P$ of a Laguerre plane. Therefore circles should become proper lines and generators should become straight lines. Moreover, in classic nearaffine planes two lines are parallel if there exists a translation which maps one line to the other. This idea should be kept for the case of Laguerre planes. But now we have only one class of generators, so the residual plane has only one class of straight lines. In the case of a Minkowski plane $\mathcal{B}$, if two points $Q, R$ determining a line $Q \triangleright R$ are given in the residual plane $\mathcal{B}[P]$, then in the initial Minkowski plane $\mathcal{B}$, generators through $P$ and $Q$ intersect also in some points $Q^{1}, Q^{2}$ and hence we have three points $Q^{1}$, $Q^{2}, R$ determining a circle. This is impossible in the case of Laguerre planes. Here the concept of the base point of a line does not seem to make sense. There are more differences. As a matter of fact, at least two classes of parallel straight lines exist in any nearaffine plane. Thus investigation of residual structures determined by Laguerre planes must really differ from the case of Minkowski planes. However, in order to get a common description of external structures concerning Laguerre planes as well as Minkowski planes, we shall use classes of parallelism to define some modification of join. Moreover, we start from two distinct families of lines: proper and straight. Since a Möbius plane has no generator, we shall not extend this idea to that case.

2. Preliminary results. The concept of an affine plane $[4, \mathrm{p} .116]$ is well known. It is a structure $(\mathbf{A}, \mathbf{L})$ with the pointset $\mathbf{A}$ provided with the family $\mathbf{L}$ of subsets of $\mathbf{A}$, called lines, such that: there exist three points not on a common line; every unordered pair of points $A, B$ uniquely determines a line $A B$ containing both points; for every line $l$ and point $P$ not on $l$, there exists a unique line $k$ with $P \in k$ and $k \cap l=\emptyset$.

We shall also use some other structures.

Let $\Pi$ be a nonempty set whose elements are called points. Let $\Sigma_{1}, \Sigma_{2}$ be families of subsets of $\Pi$, called generators, such that the following axioms hold:

(G1) Let $i \in\{1,2\}$. If $\Sigma_{i} \neq \emptyset$ then for every point $P$ there exists a unique generator $\sigma \in \Sigma_{i}$ passing through $P$.

(G2) If $\Sigma_{1} \neq \Sigma_{2}, \sigma \in \Sigma_{1}$, and $\tau \in \Sigma_{2}$, then $\sigma$ meets $\tau$ in a unique point.

We shall use the notations $\Sigma,[P]_{i}$, and $[P]$ for the union of all generators, the generator from $\Sigma_{i}$ passing through $P$, and the union of all generators through $P$, respectively (i.e. $\Sigma=\Sigma_{1} \cup \Sigma_{2} ; \sigma=[P]_{i} \Leftrightarrow \sigma \in \Sigma_{i} \wedge P \in \sigma$; and $\left.[P]=[P]_{1} \cup[P]_{2}\right)$. Distinct points $A_{1}, \ldots, A_{n}$ are said to be joinable if no generator passes through any two of them.

Let $(\Pi, \Sigma)$ be any structure satisfying $(\mathrm{G} 1),(\mathrm{G} 2)$, provided with a family $\Lambda$ of subsets of $\Pi$ called circles. 
Definition 2.1 ([7, p. 219]). The structure $\mathcal{B}=(\Pi, \Sigma, \Lambda)$ is a Benz plane if the following axioms hold:

(B1) Every circle intersects every generator in a unique point.

(B2) Through three pairwise distinct joinable points $A, B, C$, there is a unique circle $\lambda$ with $A, B, C \in \lambda$ (denoted by $(A, B, C)$ ).

(B3) Given a circle $\lambda$, a point $P \in \lambda$ and a point $Q \notin \lambda$ with $P$ and $Q$ joinable, there is a unique circle $\mu$ through $Q$ such that $\lambda \cap \mu=\{P\}$.

(B4) There is a circle $\lambda$ with at least three points such that $\Pi \backslash \lambda \neq \emptyset$.

Definition 2.2 (cf. e.g. [2], [3, p. 258], [5, pp. 45-46], [7, p. 219], [8]). A Benz plane is called a Möbius plane, a Laguerre plane or a Minkowski plane according as it contains 0,1 or 2 nonempty distinct families of generators.

By (G1), (B1) and (G2) every circle $\lambda$ of a Minkowski plane determines the following involutorial bijection $S_{\lambda}$ called the circle-symmetry with respect to $\lambda[9$, pp. 271-272].

DEFINITION 2.3.

$$
S_{\lambda}: \Pi \rightarrow \Pi, \quad S_{\lambda}(P)=Q \Leftrightarrow[P]_{1} \cap \lambda=[Q]_{2} \cap \lambda \wedge[P]_{2} \cap \lambda=[Q]_{1} \cap \lambda .
$$

There exist Minkowski planes with a point $P$ for which the following condition holds [10, conditions (A), (B), pp. 121-122]:

Let $\kappa, \lambda \in \Lambda, P \notin \kappa \cup \lambda,[P]_{i} \cap \kappa=\left\{R_{\kappa}^{i}\right\},[P]_{i} \cap \lambda=\left\{R_{\lambda}^{i}\right\}, R_{\kappa}^{i} \neq R_{\lambda}^{i}$ $(i=1,2)$.

(WA) If there exists a circle $\mu$ touching $\kappa$ at $R_{\kappa}^{1}$ and touching $\lambda$ at $R_{\lambda}^{2}$, then there also exists a circle $\nu$ touching $\kappa$ at $R_{\kappa}^{2}$ and touching $\lambda$ at $R_{\lambda}^{1}$.

(WB) If $S_{\kappa}(P) \in \lambda$ and $S_{\lambda}(P) \in \kappa$, then there exist circles $\mu, \nu$ such that

$$
\mu \cap \kappa=\left\{R_{\kappa}^{1}\right\}, \mu \cap \lambda=\left\{R_{\lambda}^{2}\right\}, \nu \cap \kappa=\left\{R_{\kappa}^{2}\right\}, \nu \cap \lambda=\left\{R_{\lambda}^{1}\right\} .
$$

Let $\Omega$ be a nonempty set, and $\Xi$ some family of subsets of $\Omega$. Elements of $\Omega$ will be called points, elements of $\Xi$, lines. Moreover, let $\triangleright: \Omega \times \Omega \backslash$ $\{(Z, Z) ; Z \in \Omega\} \rightarrow \Xi$ be a surjection called join $(\triangleright(X, Y)$ will be denoted by $X \triangleright Y)$ and let $\equiv \subset \Xi \times \Xi$ be an equivalence relation called parallelism of lines. $X$ is called the base point of the line $X \triangleright Y$. Lines satisfying the condition $X \triangleright Y=Y \triangleright X$ are called straight lines, the remaining ones, proper lines. The set of all straight lines will be denoted by $\Upsilon$.

Definition 2.4 ([11, pp. 53-54], [6, pp. 345-346]). A quadruple $(\Omega, \Xi$, $\triangleright, \equiv)$ is a nearaffine plane if the following three groups of axioms hold:

(I) Axioms of lines:

$$
\begin{aligned}
& X, Y \in X \triangleright Y \text { for all } X, Y \in \Omega, X \neq Y . \\
& Z \in X \triangleright Y \backslash\{X\} \Leftrightarrow X \triangleright Y=X \triangleright Z \text { for all } X, Y, Z \in \Omega, X \neq Y .
\end{aligned}
$$


(L3) $X \triangleright Y=Y \triangleright X=X \triangleright Z \Rightarrow X \triangleright Z=Z \triangleright X$ for all $X, Y, Z \in \Omega$, $Y \neq X \neq Z$.

(II) Axioms of parallelism:

(P1) For every line $a$ and every point $X$, there exists a unique line with base point $X$ and parallel to $a$ (we denote this line by $(X \equiv a)$ ).

(P2) $X \triangleright Y \equiv Y \triangleright X$ for all $X, Y \in \Omega, X \neq Y$.

(P3) $\quad a \equiv b \wedge a \in \Upsilon \Rightarrow b \in \Upsilon$ for all $a, b \in \Xi$.

(III) Axioms of richness:

(N1) There exist at least two nonparallel straight lines.

(N2) Every line $a$ meets every straight line $b$ nonparallel to $a$ in exactly one point.

Definition 2.5. For any Minkowski plane $\mathcal{B}=(\Pi, \Sigma, \Lambda)$ with a distinguished point $P$ we put:

- $\Omega=\Pi \backslash[P], \Xi=\{\sigma \cap \Omega ; \sigma \in \Sigma, P \notin \sigma\} \cup\left\{\lambda \cap \Omega \cup\left\{S_{\lambda}(P)\right\} ; \lambda \in \Lambda\right.$, $P \notin \lambda\}$.

- $\equiv \subset \Xi \times \Xi$ and $a \equiv b$ if one of the following mutually exclusive conditions holds:

$-a=b$;

- $a=\sigma \cap \Omega$ and $b=\tau \cap \Omega$ for some $\sigma, \tau \in \Sigma$ with $\sigma \cap \tau=\emptyset$;

- $a=\lambda \cap \Omega \cup\left\{S_{\lambda}(P)\right\}$ and $b=\mu \cap \Omega \cup\left\{S_{\mu}(P)\right\}$ for some $\lambda, \mu \in \Lambda$ such that $S_{\lambda}(P) \neq S_{\mu}(P), S_{\lambda}(P) \in\left[S_{\mu}(P)\right]$ and $\lambda \cap \mu \cap \Omega=\emptyset$;

- $a=\lambda \cap \Omega \cup\left\{S_{\lambda}(P)\right\}, b=\mu \cap \Omega \cup\left\{S_{\mu}(P)\right\}$, and $S_{\lambda}(P) \notin\left[S_{\mu}(P)\right]$ for some $\lambda, \mu \in \Lambda$, and there exists $\nu \in \Lambda$ such that $S_{\lambda}(P), S_{\mu}(P) \in$ $\left[S_{\nu}(P)\right]$ and $\lambda \cap \nu \cap \Omega=\emptyset=\nu \cap \mu \cap \Omega$;

- $\triangleright: \Omega \times \Omega \backslash\{(Z, Z) ; Z \in \Omega\} \rightarrow \Xi$ and

- $X \triangleright Y=\sigma \cap \Omega$ if $X, Y \in \sigma$ for some $\sigma \in \Sigma$;

- $X \triangleright Y=\lambda \cap \Omega \cup\{X\}$ if $X \notin[Y],[X] \cap[P]=\{U, V\}$, and $\lambda=(U, V, Y)$ (then $\left.S_{\lambda}(P)=X\right)$.

Theorem 2.1 ([10, pp. 120-123]). If a Minkowski plane $\mathcal{B}=(\Pi, \Sigma, \Lambda)$ with a distinguished point $P$ satisfies condition $(\mathrm{W})$, then the structure $\mathcal{B}[P]=(\Omega, \Xi, \triangleright, \equiv)$ given in Definition 2.5 is a nearaffine plane.

3. Pseudo-affine planes. Let $\Omega$ be a nonempty set of points provided with two disjoint families $\Psi, \Upsilon$ of subsets of $\Omega$ called proper lines and straight lines, respectively. We define

$$
\Gamma=\left\{(Q, R) \in \Omega \times \Omega ; \bigvee_{l \in \Upsilon} Q \in l \wedge R \in l\right\} .
$$

Further we require: 
(i) (a) $\Upsilon=\Upsilon_{1} \cup \Upsilon_{2}, \Upsilon_{1} \neq \emptyset \neq \Upsilon_{2}$, and either $\Upsilon_{1}=\Upsilon_{2}$ or $\Upsilon_{1} \cap \Upsilon_{2}=\emptyset$.

(b) If $\Upsilon_{1} \cap \Upsilon_{2}=\emptyset$ then every $p \in \Upsilon_{1}$ meets every $q \in \Upsilon_{2}$ in a unique point.

(c) For every point $A$ there exists a unique $p \in \Upsilon_{i}(i=1,2)$ such that $A \in p$.

(ii) For $i \in\{1,2\}$ an equivalence relation $\simeq_{i} \subset \Psi \times \Psi$ is given and $\Upsilon_{1}=\Upsilon_{2} \Leftrightarrow \simeq_{1}=\simeq_{2}$. We define $\Theta_{i}=\left\{[l]_{\simeq_{i}} ; l \in \Psi\right\}, \Theta=\Theta_{1} \cup \Theta_{2}$ and $\simeq=\simeq_{1} \cup \simeq_{2}$. The relation $\simeq$ is called pseudo-parallelism.

(iii) There exists a surjective mapping $\triangle:[(\Omega \times \Omega) \backslash \Gamma] \times \Theta \rightarrow \Psi$ called pseudo-join.

We will consider a couple of structures in this paper. In order not to mix the structures and their blocks, we shall denote:

- points (of all structures considered) by capital Latin letters $A, B$, $C, \ldots$;

- lines (of any kind) by small Latin letters $a, b, c, \ldots$;

- classes of pseudo-parallelism by small letters of the first part of the Greek alphabet, $\alpha, \beta, \gamma, \ldots$;

- the image of $(A, B, \alpha)$ with respect to $\triangle$ by $\triangle(A, B, \alpha)$;

- circles of a Benz plane by small letters of the middle part of the Greek alphabet, $\kappa, \lambda, \mu, \nu, \ldots$;

- generators of a Benz plane by small letters of the last part of the Greek alphabet, $\sigma, \varsigma, \tau, \ldots$

Definition 3.1. The structure $(\Omega, \Psi, \Upsilon, \simeq, \triangle)$ is called a pseudo-affine plane if the following axioms hold:

(PA0) There exists $p \in \Upsilon$ with two distinct points and $\Omega \backslash p \neq \emptyset$.

(PA1) $\quad A, B \in \triangle(A, B, \alpha) \wedge \triangle(A, B, \alpha) \in \alpha$.

(PA2) $\quad C, D \in \triangle(A, B, \alpha) \wedge C \neq D \Rightarrow \triangle(A, B, \alpha)=\triangle(C, D, \alpha)$.

(PA3) Every straight line intersects every proper line in a unique point.

(PA4) For every $a \in \Psi$ and every $A \notin a$ there exists a unique $b \in \Psi$ such that $b \simeq_{i} a(i=1,2), A \in b$, and $a \cap b=\emptyset$.

Corollary 3.1. (a) $\triangle(A, B, \alpha)=\triangle(B, A, \alpha)$.

(b) If $a, b, c \in \alpha$ and $a \cap b=\emptyset=b \cap c$, then $a \cap c=\emptyset$.

(c) $\triangle(A, B, \alpha) \in \beta \Rightarrow \triangle(A, B, \alpha)=\triangle(A, B, \beta)$.

Proof. (a) follows immediately from (PA1) and (PA2) (we put $C=B$, $D=A$ ), and (b) follows immediately from (PA4). Let $\triangle(A, B, \alpha) \in \beta$. By (PA1) we have $\triangle(A, B, \alpha) \in \alpha \cap \beta$. Since $\simeq_{i}(i=1,2)$ is an equivalence relation, $\triangle(A, B, \alpha)$ belongs to exactly one class of $\Theta_{i}$. Thus we obtain $\alpha=\beta$ if $\alpha, \beta \in \Theta_{1}$ or $\alpha, \beta \in \Theta_{2}$. Let $\alpha \neq \beta$. Then $\Theta_{1} \neq \Theta_{2}\left(\right.$ so $\left.\simeq_{1} \neq \simeq_{2}\right)$ and e.g. $\alpha \in \Theta_{1}, \beta \in \Theta_{2}$. The elements $A, B$ and $\beta$ uniquely determine the 
image $\triangle(A, B, \beta)$. By (PA1), it must be the proper line containing $A, B$ and belonging to $\beta$, i.e. $\triangle(A, B, \alpha)$.

TheOREM 3.1. Let $(\Omega, \Psi, \Upsilon, \simeq, \triangle)$ be a pseudo-affine plane and let $\alpha \in \Theta$. Then $(\Omega, \Upsilon \cup \alpha)$ is an affine plane.

Proof. Let $A, B \in \Omega, A \neq B$. If $(A, B) \in \Gamma$ then there exists $p \in \Upsilon$ such that $A, B \in p$ and, by requirement (i), such a $p$ is unique. By (PA3), no $q \in \Psi$ contains both $A$ and $B$. If $(A, B) \notin \Gamma$ then $\triangle(A, B, \alpha)$ uniquely determined by (iii) is the unique element of $\Upsilon \cup \alpha$ containing $A, B$.

Let $p \in \Upsilon \cup \alpha$ and $A \in \Omega \backslash p$. If $p \in \Upsilon_{1}$ then there exists a unique $q \in \Upsilon_{1}$ such that $A \in q$ and $q \cap p=\emptyset$ by (i)(c). Every $r \in \Psi$ meets $p$ by (PA3) and the same arguments work for $r \in \Upsilon_{2}$ if $\Upsilon_{1} \neq \Upsilon_{2}$.

If $p \in \alpha$ then $p \cap r \neq \emptyset$ for every $r \in \Upsilon$ by (PA3), and (PA4) shows that there exists a unique $q \in \alpha$ such that $A \in q$ and $p \cap q=\emptyset$.

(PA0) implies that there exist three points not on a common line from $\Upsilon \cup \alpha$.

Definition 3.2. For any Laguerre or Minkowski plane $\mathcal{B}=(\Pi, \Sigma, \Lambda)$ and $P \in \Pi$ we put

(a) $\Omega=\Pi \backslash[P]$;

(b) $\Upsilon=\{\sigma \cap \Omega ; \sigma \in \Sigma, P \notin \sigma\}$;

(c) for every $\lambda \in \Lambda$ with $P \notin \lambda$,

$$
\begin{aligned}
\lambda^{*} & = \begin{cases}\lambda \cap \Omega & \text { if } \mathcal{B} \text { is a Laguerre plane, } \\
(\lambda \cap \Omega) \cup\left\{S_{\lambda}(P)\right\} & \text { if } \mathcal{B} \text { is a Minkowski plane, }\end{cases} \\
\Psi & =\left\{\lambda^{*} ; \lambda \in \Lambda, P \notin \lambda\right\} ;
\end{aligned}
$$

(d) $\lambda^{*} \simeq \mu^{*} \Leftrightarrow \lambda \cap \mu \cap[P] \neq \emptyset$.

Using (B1) we get:

Corollary 3.2. Let $\simeq$ be the relation given in Definition $3.2(\mathrm{~d})$. If $\mathcal{B}$ is a Laguerre plane then $\simeq$ is an equivalence relation. If $\mathcal{B}$ is a Minkowski plane then $\simeq=\simeq_{1} \cup \simeq_{2}$, where $\lambda^{*} \simeq_{i} \mu^{*} \Leftrightarrow \lambda \cap \mu \cap[P]_{i} \neq \emptyset(i=1,2)$ and $\simeq_{1}, \simeq_{2}$ are equivalence relations.

Let $\Theta$ denote the set of all equivalence classes of the relation $\simeq$ from Definition 3.2(d) (i.e. in the case of Minkowski planes we have $\Theta=\Theta_{1} \cup \Theta_{2}$, where $\Theta_{i}$ is the set of all classes of $\left.\simeq_{i}, i=1,2\right)$. In both cases there is a

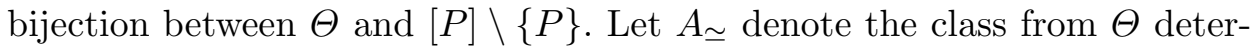
mined by $A \in[P] \backslash\{P\}$. Preserving all notations from Definition 3.2 and the meaning of $\Gamma$ we make

Definition 3.3. $\triangle:[(\Omega \times \Omega) \backslash \Gamma] \times \Theta \rightarrow \Psi, \triangle(Q, R, \alpha)=\lambda^{*}$, where $\alpha=A_{\simeq \text { for some } A \in[P] \backslash\{P\} \text { and we put }}$ 
(a) $\lambda=(Q, R, A)$ for joinable $Q, R, A$;

(b) $\lambda=(Q, A, B)$, where $\{B\}=[R]_{2} \cap[P]_{1}$ for $[R]_{1}=[A]_{1}$;

(c) $\lambda=(Q, A, B)$, where $\{B\}=[R]_{1} \cap[P]_{2}$ for $[R]_{2}=[A]_{2}$.

Corollary 3.3. In cases (b), (c) of Definition 3.3 we obtain $S_{\lambda}(P)=R$.

TheOREM 3.2. The structure $\mathcal{B}\langle P\rangle=(\Omega, \Psi, \Upsilon, \simeq, \triangle)$ given in Definitions 3.2 and 3.3 is a pseudo-affine plane.

Proof. We have to verify requirements (i)-(iii) and axioms (PA0)-(PA4). Corollary 3.2 ensures the existence of $\simeq_{i}(i=1,2)$. If $\mathcal{B}$ is a Laguerre plane then we obviously obtain $\Upsilon_{1}=\Upsilon_{2}=\Upsilon$ and $\simeq_{1}=\simeq_{2}=\simeq$. If $\mathcal{B}$ is a Minkowski plane then $\Upsilon_{1} \cap \Upsilon_{2}=\emptyset$ and $\simeq_{1} \neq \simeq_{2}$. Thus (i)(a) and (ii) hold. (G1) and (G2) yield (i) (c) and (i)(b), respectively. By Definition 3.3, for every triple $(Q, R, \alpha) \in[(\Omega \times \Omega) \backslash \Gamma] \times \Theta$ the line $\triangle(Q, R, \alpha)$ is uniquely determined. We shall show that $\triangle$ is surjective. If $a \in \Psi$ then $a=\lambda^{*}$ for some $\lambda \in \Lambda$ with $P \notin \lambda$. By (B1) there exists $A \in([P] \backslash\{P\}) \cap \lambda$, and by (B4), there exist at least two points $Q, R$ (resp. one point $Q$ ) in $\lambda \cap \Omega$ if $\mathcal{B}$ is a Laguerre (resp. Minkowski) plane. Thus we obtain $a=\triangle\left(Q, R, A_{\simeq}\right)$ (resp. $a=\triangle\left(Q, S_{\lambda}(P), A_{\simeq}\right)$ ).

In order to check (PA0)-(PA4), let us use Definition 3.3 and assume $a=\triangle(A, B, \alpha)=\triangle\left(A, B, Q_{\simeq}\right)=\lambda^{*}$, where $Q \in([P] \backslash\{P\}) \cap \lambda$. We have the following three exclusive possibilities for $A, B: A, B \in \lambda ; A=S_{\lambda}(P)$, $B \in \lambda ; B=S_{\lambda}(P), A \in \lambda$.

(PA0) is immediate from (B4), (G1) and (B1).

(PA1): $Q \in([P] \backslash\{P\}) \cap \lambda$ with $Q_{\simeq}=\alpha$ means $\lambda^{*} \in \alpha$. In all three possibilities mentioned above we get $A, B \in \lambda^{*}$.

(PA2): Let $C, D \in \triangle(A, B, Q \simeq), C \neq D,\{C, D\} \neq\{A, B\}$. If $C, D \in \lambda$ then $\lambda=(C, D, Q)$ and $\lambda^{*}=\triangle(C, D, Q \simeq)$. If $A=S_{\lambda}(P)=C$ then $B, D \in \lambda$ and for $R \in[P] \cap \lambda, R \neq Q$ we have $(B, Q, R)=\lambda=(D, Q, R)$. Thus $\triangle\left(A, B, Q_{\simeq}\right)=\lambda^{*}=\triangle\left(C, D, Q_{\simeq}\right)$.

(PA3): In the case of Laguerre planes straight and proper lines are unchanged generators and circles without one point, respectively, not through $P$. So (PA3) and (B1) coincide. In the case of Minkowski planes every circle $\lambda$ not through $P$ meets every generator not through $S_{\lambda}(P)$ in a point not in $[P]$. Thus the point of intersection is on both traces, i.e. on the proper line and on the straight line. Finally, the traces of $\left[S_{\lambda}(P)\right]_{+}$and of $\left[S_{\lambda}(P)\right]_{-}$intersect $\lambda^{*}$ in $S_{\lambda}(P)$.

(PA4): Let $C \notin a ; b \in \Psi ; a \neq b=\mu^{*}$ for some $\mu \in \Lambda, P \notin \mu$. By Definition 3.2(d), $b \simeq a$ if there exists $R \in[P] \cap \lambda \cap \mu$. If $R \neq T \in[P] \cap \lambda \cap \mu$ (i.e. $a \simeq_{i} b$ for $i \in\{1,2\}$ in a Minkowski plane) then $S_{\lambda}(P) \in \lambda^{*} \cap \mu^{*}$, hence $a \cap b \neq \emptyset$. If $[P] \cap \lambda \cap \mu=\{R\}$ then either $\lambda \cap \mu=\{R\}$ or there exists $T \in \lambda \cap \mu \backslash[P]$. In the former case, by (B3), exactly one such $\mu$ passes through $C$. In the latter case $T \in \lambda^{*} \cap \mu^{*}$, i.e. $a \cap b \neq \emptyset$. 
Definition 3.4. For every $(A, B) \notin \Gamma$ we put

$$
[A, B]=\{A, B\} \cup\left\{C \in \Omega ; \neg \bigvee_{l \in \Psi} A, B, C \in l\right\} .
$$

Theorem 3.3. Let $\mathcal{B}=(\Pi, \Sigma, \Lambda)$ be a Laguerre or Minkowski plane, $P \in \Pi$ and $[A, B]$ given in the pseudo-affine plane $\mathcal{B}\langle P\rangle$. Then in the initial Benz plane $\mathcal{B}$ we have $[A, B] \cup\{P\}=(A, B, P)$.

Proof. Clearly, $A, B, P \in([A, B] \cup\{P\}) \cap(A, B, P)$ so let $C \notin\{A, B, P\}$. Suppose $C \in(A, B, P)$ and $C \notin[A, B]$. Hence $(A, B, P)=(A, B, C)$ and there exists some $\alpha \in \Theta$ such that $C \in \triangle(A, B, \alpha)$. But $\alpha=Q \simeq$ for some $Q \in[P] \backslash\{P\}$. Thus $(A, B, P)=(A, B, C)=(A, B, Q)$, which contradicts (B1). Conversely, let $C \in[A, B]$ and suppose $C \notin(A, B, P)$. Then $P \notin$ $(A, B, C)$ and by (B1), there exists some point $Q \in[P] \backslash\{P\}$ such that $Q \in$ $(A, B, C)$. But $Q$ determines the class $\alpha=Q_{\simeq}$ and $(A, B, C)^{*}=\triangle(A, B, \alpha)$. So $C \notin[A, B]$, a contradiction.

Corollary 3.4. The plane $\mathcal{B}\langle P\rangle$ completely determines the plane $\mathcal{B}$.

4. A connection with nearaffine planes. Among all pseudo-affine planes $\mathcal{P} \mathcal{A}=(\Omega, \Psi, \Upsilon, \simeq, \triangle)$ with $\Upsilon=\Upsilon_{1} \cup \Upsilon_{2}, \Upsilon_{1} \neq \Upsilon_{2}$, we distinguish the class satisfying the following additional axioms (PV) and (PW).

(PV) There exist bijections $f_{i}: \Theta_{i} \rightarrow \Upsilon_{i}(i=1,2)$ such that $f_{1}\left(\alpha_{1}\right) \cap$ $f_{2}\left(\alpha_{2}\right) \subset p$ for any $\alpha_{i} \in \Theta_{i}$ and $p \in \alpha_{1} \cap \alpha_{2}$.

Definition 4.1. $f: \Theta_{1} \times \Theta_{2} \rightarrow \Omega$ and $f\left(\alpha_{1}, \alpha_{2}\right)=Q \Leftrightarrow f_{i}\left(\alpha_{i}\right)=q_{i}$, where $q_{i} \in \Upsilon_{i}$ and $Q \in q_{i}$ for $i=1,2$.

Corollary 4.1. If a pseudo-affine plane $\mathcal{P} \mathcal{A}=(\Omega, \Psi, \Upsilon, \simeq, \triangle)$ satisfies $(\mathrm{PV})$ then every ordered pair of points $(Q, R) \notin \Gamma$ uniquely determines $l \in \Psi$ such that $Q, R \in l$.

Proof. By (PV), $Q=f(\alpha, \beta)$ for some $(\alpha, \beta) \in \Theta_{1} \times \Theta_{2}$. Thus we obtain $l=\triangle(Q, R, \alpha)=\triangle(Q, R, \beta)$.

Definition 4.2. $Q$ is called a base point of a proper line $l$ if $l \in \alpha \cap \beta$ and $Q=f(\alpha, \beta)$ for some $(\alpha, \beta) \in \Theta_{1} \times \Theta_{2}$. Every point of a straight line is called its base point.

Corollary 4.2. Every proper line $p=\triangle\left(Q, R, \alpha_{i}\right)$ with $\alpha_{i} \in \Theta_{i}(i=$ $1,2)$ has its base point on the straight line $q_{i} \in \Upsilon_{i}$ if and only if $f_{i}\left(\alpha_{i}\right)=q_{i}$.

Now consider

(PW) If $\alpha_{i}, \beta_{i} \in \Theta_{i} ; \alpha_{i} \neq \beta_{i}$ for $i \in\{1,2\} ; a \in \alpha_{1} \cap \alpha_{2} ; b \in \alpha_{1} \cap \beta_{2}$; $c \in \beta_{1} \cap \beta_{2} ; a \cap b=\emptyset=b \cap c ; d \in \beta_{1} \cap \alpha_{2} ; e \in \beta_{1} \cap \beta_{2}$ and $a \cap d=\emptyset=d \cap e$ then $c=e$. Moreover, if $f\left(\beta_{1}, \beta_{2}\right) \in a$ then $f\left(\alpha_{1}, \alpha_{2}\right) \in c$. 
Definition 4.3. For any pseudo-affine plane $(\Omega, \Psi, \Upsilon, \simeq, \triangle)$ satisfying $(\mathrm{PV})$ and $(\mathrm{PW})$ we define the structure $(\Omega, \Xi, \equiv, \triangleright)$ as follows:

(i) $\Xi=\Psi \cup \Upsilon$.

(ii) $\equiv \subset \Xi \times \Xi$ and $a \equiv b$ if one of the following mutually exclusive conditions holds:

(a) $a=b$;

(b) $a, b \in \Upsilon$ and $a \cap b=\emptyset$;

(c) $a, b \in \Psi, a \cap b=\emptyset$ and $a \simeq_{1} b \vee a \simeq_{2} b$;

(d) $a, b \in \Psi, a \neq b$ and there exists $c \in \Psi$ such that $a \cap c=\emptyset=c \cap b$ and $\left(a \simeq_{1} c \wedge c \simeq_{2} b\right) \vee\left(a \simeq_{2} c \wedge c \simeq_{1} b\right)$.

(iii) $\triangleright: \Omega \times \Omega \backslash\{(Z, Z) ; Z \in \Omega\} \rightarrow \Xi$, and if $(X, Y) \in \Gamma$ then $X \triangleright Y=$ $l$, where $l \in \Upsilon$ and $X, Y \in l$, while if $(X, Y) \notin \Gamma$ then $X \triangleright Y$ is the proper line containing $X, Y$ and uniquely determined by Corollary 4.1.

In the structure $(\Omega, \Xi, \equiv, \triangleright)$ from Definition 4.3 we have $\Xi=\Psi \cup \Upsilon$ and we still call elements of $\Xi, \Psi$ and $\Upsilon$ lines, proper lines and straight lines, respectively.

TheOREM 4.1. The structure $(\Omega, \Xi, \equiv, \triangleright)$ given in Definition 4.3 is a nearaffine plane.

Proof. (I) Clearly, the definition of the mapping $\triangleright$ is correct. It remains to show that $\triangleright$ is surjective. Let $l \in \Xi$. If $l \in \Upsilon$ then $l=X \triangleright Y$ for any $X, Y$ with $X \neq Y$. If $l \in \Psi$ then $l=\triangle(U, V, \alpha)=\triangle(U, V, \beta)$ for some $(U, V) \in \Omega \times \Omega \backslash \Gamma$ and $\alpha \in \Theta_{1}, \beta \in \Theta_{2}$. By (PV) and Definition 4.1, there exists $Q \in l$ with $f(\alpha, \beta)=Q$. If $Q \neq U$ then we obtain $l=Q \triangleright U$. If $Q=U$ then $Q \neq V$ and $l=Q \triangleright V$.

(II) $\equiv$ is an equivalence relation. Indeed, it is immediate from Definition $4.3(\mathrm{ii})$ that $\equiv$ is reflexive and symmetric. To show transitivity, assume that $a \equiv b$ and $b \equiv c$. Then either $a, b, c \in \Upsilon$ or $a, b, c \in \Psi$. The former case is obvious, since then $a, b, c \in \Upsilon_{1}$ or $a, b, c \in \Upsilon_{2}$. Any two lines $p, q$ from $\Upsilon_{i}(i=1,2)$ are disjoint or equal, i.e. $p \equiv q$, by Definition 4.3(ii)(a), (ii)(b). Consider the latter case and assume $a \neq b \neq c$. We shall consider the following possibilities, omitting cases obtained by exchanging $\simeq_{1}$ and $\simeq_{2}$.

(A) $a \simeq_{1} b \simeq_{1} c$ and $a \cap b=\emptyset=b \cap c$. By (ii), $\simeq_{1}$ is an equivalence relation, so $a \simeq_{1} c$. In view of Corollary $3.1(\mathrm{~b}), a \cap c=\emptyset$. Therefore $a \equiv c$ (Definition 4.3(ii)(c)).

(B) $a \simeq_{1} b \simeq_{2} c$ and $a \cap b=\emptyset=b \cap c$. Then we get item (ii)(d) of Definition 4.3 .

(C) $a \simeq_{1} b, a \cap b=\emptyset$ and there exists $d \in \Psi$ with $b \simeq_{1} d \simeq_{2} c$ and $b \cap d=\emptyset=d \cap c$. Using (A) for $a, b, d$ we get $a \simeq_{1} d, a \cap d=\emptyset$. Now we have $a \simeq_{1} d \simeq_{2} c$ and using (B) we get $a \equiv c$ (Definition 4.3(ii)(d)). 
(D) $a \simeq_{1} b, a \cap b=\emptyset$ and there exists $d \in \Psi$ such that $b \simeq_{2} d \simeq_{1} c$ and $b \cap d=\emptyset=d \cap c$. We have $a, b \in \alpha ; b, d \in \beta ; d, c \in \gamma$ for some $\alpha, \gamma \in \Theta_{1}$, $\beta \in \Theta_{2}$. But also $c \in \delta$ for some $\delta \in \Theta_{2}$. Consider the lines $b, d, c$. Set $T=f(\alpha, \delta)$. By (PA4), there exists a unique $e \in \alpha$ such that $T \in e$ and $e \cap b=\emptyset$. In view of Corollary 4.2, the base point of $e$ is on the straight line $f_{1}(\alpha)$. Since $e$ intersects $f_{1}(\alpha)$ in exactly one point, $T$ must be the base point of $e$. Therefore $e \in \delta$. Let $S$ be the base point of $c$. Using (PA4) again, there exists a unique $k \in \delta$ such that $S \in k$ and $k \cap e=\emptyset$. So we obtain $b \simeq_{1} e \simeq_{2} k, b \cap e=\emptyset=e \cap k$ and $b \simeq_{2} d \simeq_{1} c, b \cap d=\emptyset=d \cap c$. By $(\mathrm{PW})$, we get $k=c$, i.e. $e \cap c=\emptyset$. Therefore we have got $a \simeq_{1} b \simeq_{1} e \simeq_{2} c$, $a \cap b=\emptyset=b \cap e=e \cap c$ and now it suffices to use (C).

(E) There exist lines $d, e$ with $a \simeq_{1} d \simeq_{2} b, a \cap d=\emptyset=d \cap b, b \simeq_{2} e \simeq_{1} c$, and $b \cap e=\emptyset=e \cap c$. Then we get $a \simeq_{1} d \simeq_{2} e \simeq_{1} c$, i.e. (D).

(F) There exist lines $d$, e with $a \simeq_{1} d \simeq_{2} b, a \cap d=\emptyset=d \cap b, b \simeq_{1} e \simeq_{2} c$, and $b \cap e=\emptyset=e \cap c$. Since $a \simeq_{1} d \simeq_{2} b \simeq_{1} e$, we can first use the arguments from (D) to get a line $k$ with $a \simeq_{1} k \simeq_{2} e$. Now we have $c \simeq_{2} e \simeq_{2} k \simeq_{1} a$ and use arguments from $(\mathrm{C})$ to get $c \equiv a$.

(III) Verification of axioms (L1)-(N2).

(L1) is obvious. Also (L2) and (L3) are obvious for straight lines $X \triangleright Y$. So assume $X \triangleright Y=\triangle(X, Y, \alpha)=\triangle(X, Y, \beta)$, where $X=f(\alpha, \beta)$.

(L2): Let $Z \in X \triangleright Y \backslash\{X\}$. Then $Z \in \triangle(X, Y, \alpha)$ for some $\alpha \in \Theta$ and (PA2) implies $\triangle(X, Y, \alpha)=(X, Z, \alpha)=X \triangleright Z$.

By (PA1), $Z \in \triangle(X, Z, \alpha)$. $X \triangleright Z$ is defined only for $Z \neq X$, and $X \triangleright Y=$ $X \triangleright Z$ means $\triangle(X, Y, \alpha)=\triangle(X, Z, \alpha)$, so $Z \in \triangle(X, Y, \alpha) \backslash\{X\}$.

(L3): Clearly, $Y=f(\gamma, \delta)$ for some $(\gamma, \delta) \in \Theta_{1} \times \Theta_{2}$. The assumption $X \triangleright Y=Y \triangleright X=X \triangleright Z$ gives $\triangle(X, Y, \alpha)=\triangle(Y, X, \gamma)=\triangle(X, Z, \alpha)$. Every proper line belongs to exactly one class from $\Theta_{i}, i=1,2$. Thus $\alpha=\gamma$. Similarly we obtain $\beta=\delta$. Hence $X=f(\alpha, \beta)=f(\gamma, \delta)=Y$. Therefore $X \triangleright Y=Y \triangleright X$ is impossible for proper lines.

(P3), (N2), (N1): By Definition 4.3(ii), if $a$ is a proper line and $b$ is a straight line then $a \not \equiv b$. Thus we obtain (P3). As (N2) and (PA3) coincide, we get (N2). Since $\Upsilon_{1}$ and $\Upsilon_{2}$ are two distinct classes of straight lines, we also get (N1).

(P1): Let $a \in \Xi=\Psi \cup \Upsilon, X \in \Omega, X=f(\alpha, \beta)$ for some $(\alpha, \beta) \in \Theta_{1} \times \Theta_{2}$. For straight lines $a, b$ we have $a \equiv b \Leftrightarrow a \simeq b \Leftrightarrow(a \cap b=\emptyset \vee a=b)$. Thus if $a \in \Upsilon_{i}$ then $(X \equiv a)$ is the straight line from $\Upsilon_{i}$ passing through $X$ (see requirement (i)(c)). Let $a \in \Psi$. Then $a=U \triangleright V=\triangle(U, V, \gamma)=\triangle(U, V, \delta)$ for some $(U, V) \notin \Gamma$ and $(\gamma, \delta) \in \Theta_{1} \times \Theta_{2}$, where $U=f(\gamma, \delta)$. If $X=U$ then $(X \equiv a)=a$ and then $a \not \equiv b$ for every line $b$ different from $a$ and passing through $X$, by Definition 4.3(ii) and Corollary 4.2. Assume that $X \neq U$. If $X, U$ are on a common straight line from $\Upsilon_{1}$ then $X \notin a$, and by (PA4), there exists a unique proper line $b$ through $X$ with $a \cap b=\emptyset$ and $b \in \gamma$. By 
Definition 4.3(ii)(c), $b \equiv a$. By Corollary 4.2, the base point of $b$ lies on the straight line from $\Upsilon_{1}$ passing through $U$ and by (PA3), it must be $X$. Then obviously $\gamma=\alpha$, i.e. $b \in \alpha \cap \beta$. Let $X, U$ be not on a common straight line and write $\{Y\}=u_{1} \cap x_{2}$, where $u_{1} \in \Upsilon_{1}, U \in u_{1}, x_{2} \in \Upsilon_{2}$, and $X \in x_{2}$. As before, we get uniquely determined proper lines $b$ and $c$ with base points $Y$ and $X$, respectively, such that $b \in \gamma \cap \beta, b \cap a=\emptyset, c \in \alpha \cap \beta$, and $c \cap b=\emptyset$. Definition $4.3(\mathrm{ii})(\mathrm{d})$ implies that $a \equiv c$ and that the only other way to get a line $e$ with base point $X$ and $e \equiv a$ is the following: Let $\{Z\}=u_{2} \cap x_{1}$ (notations as before) and take proper lines $d$, $e$ such that $Z$ is the base point of $d$ and $X$ is the base point of $e$ (then $d \in \alpha \cap \delta, e \in \alpha \cap \beta), d \cap a=\emptyset$, and $e \cap d=\emptyset$. By $(\mathrm{PW}), e=c$.

(P2): If $X \triangleright Y$ is a straight line then $X \triangleright Y=Y \triangleright X$ and so $X \triangleright Y \equiv Y \triangleright X$, by Definition 4.3(ii)(a). Let $a=X \triangleright Y$ be proper. Checking (P1), we have already shown that there exists a unique proper line $b$ with base point $Y$ such that $X \triangleright Y \equiv b$. There exist some $\alpha, \gamma \in \Theta_{1}$ and $\beta, \delta \in \Theta_{2}$ such that $X=f(\alpha, \beta)$ and $Y=f(\gamma, \delta)$. Clearly, there also exists a line $c$ with $a \cap c=\emptyset=c \cap b$ and $c \in \alpha \cap \delta$. Since $Y \in a$ and the last part of (PW) is valid, we obtain $X \in b$.

\section{References}

[1] J. André, Some new results on incidence structures, in: Colloquio Internazionale sulle Teorie Combinatori, Vol. II, Atti dei Convegni Lincei 17, 1976, 201-222.

[2] W. Benz, Vorlesungen über Geometrie der Algebren, Springer, Berlin, 1973.

[3] Y. Chen, A characterization of some geometries of chains, Canad. J. Math. 26 (1974), 257-272.

[4] P. Dembowski, Finite Geometries, Springer, Berlin, 1968.

[5] W. Heise und H. Seybold, Das Existenzproblem der Möbius-, Laguerre- und Minkowski-Erweiterungen endlicher affiner Ebenen, Sitz. Ber. Bayer. Akad. Wiss. Math.Nat. Kl. 1975, 43-58.

[6] J. Jakóbowski, Nearaffine planes related to pseudo-ordered fields, Bull. Polish Acad. Sci. Math. 50 (2002), 345-360.

[7] H. J. Kroll, Anordnungsfragen in Benz-Ebenen, Abh. Math. Semin. Univ. Hamburg 46 (1977), 217-255.

[8] R. Löwen and R. U. Pfüller, Two-dimensional Laguerre planes over convex functions, Geom. Dedicata 23 (1987), 73-85.

[9] N. Percsy, Finite Minkowski planes in which every circle-symmetry is an automorphism, ibid. 10 (1981), 269-282.

[10] H. A. Wilbrink, Finite Minkowski planes, Geom. Dedicata 12 (1982), 119-129.

[11] —, Nearaffine planes, ibid. 12 (1982), 53-62.

Jan Jakóbowski

University of Warmia and Mazury in Olsztyn

Faculty of Mathematics and Informatics

Żołnierska 14, 10-561 Olsztyn, Poland

E-mail: jjakob@matman.uwm.edu.pl 